\title{
Quasi-one dimensional graphite ribbon structures in the presence of a magnetic field and the on-site Coulomb correlation at half-filling
}

\author{
Jayeeta Chowdhury ${ }^{1}$, Shreekantha $\mathrm{Sil}^{2}$, S. N. Karmakar ${ }^{3}$, Bibhas \\ Bhattacharyya $^{4,5}$ \\ ${ }^{1}$ Department of Physics, Scottish Church College, 1 \& 3 Urquhart Square, \\ Kolkata 700 006, India \\ ${ }^{2}$ Department of Physics, Visva-Bharati, Santiniketan, West Bengal, 731235, India \\ ${ }^{3}$ TCMP Division, Saha Institute of Nuclear Physics, 1/AF Bidhannagar, Kolkata \\ 700 064, India \\ ${ }^{4}$ Department of Physics and Astrophysics, West Bengal State University, \\ Barasat, Kolkata 700 126, India \\ ${ }^{5}$ Department of Physics, Jadavpur University, Kolkata 700 032, India
}

Shortened version of the title:

Graphite ribbons in presence of magnetic field and Hubbard correlation 


\begin{abstract}
We have presented the role of the Coulomb interaction $(U)$ and the magnetic field $(\vec{B})$ on the ground state properties of the quasi-one dimensional graphite ribbon structures at half-filling. Mean field Hartree-Fock Approximation is used to study the systems. To understand the boundary effects in graphite structures, we have compared the results of these systems with those of the square lattice ribbon structures. Studying the density of states, the Drude weight and the charge gap, we have drawn the $U-B$ phase diagrams for the zigzag and the armchair graphite ribbons.
\end{abstract}




\subsection{Introduction}

Recently, there has been a lot of theoretical work on the carbon-network structures due to the scope of wide applicability of the materials composed of carbon atoms in nanotechnology devices [1]. Study of nanoscale graphites seems interesting since in these systems edge and bulk effects are comparable. States near the Fermi level have strikingly different features depending on the boundary geometry of the graphites. The graphite lattices with finite widths, known as graphite ribbons, are the simplest systems to study the boundary effects. There are two basic edge shapes in graphites, the armchair and the zigzag. Zigzag ribbons posses localized edge states at the Fermi level, which correspond to the non-bonding molecular orbital [2, 3, 4]. These types of edge states are completely absent in armchair ribbons. It was observed, both experimentally and theoretically, that depending on the boundary geometry and size, graphite ribbons may be metallic or insulating [5, 6, 7, 8, 9, 10]. In the recent past, there has been a lot of exciting experimental work on the graphenes exploring their response to electric and magnetic fields [11, 12].

A lot of work has been done studying the magnetic properties of the graphite ribbons [13, 14, 15, 16]. It was observed that an external magnetic field can induce in these systems several features such as metal-insulator transition [13], Aharonov-Bohm oscillation of conductance [16] etc. Inclusion of the Zeeman interaction results in a step-like structure of the magnetoconductance [15] in graphite systems. On the other hand, electronic properties of the graphite ribbons in the presence of the on-site Coulomb repulsion are also studied using the Hubbard model within the Hartree-Fock Approximation [3, 17, 18; ; the Hubbard interaction is found to favor the formation of ferrimagnetic spin polarization along the edge of the zigzag ribbons. Vacancy induced magnetism in graphene ribbons has also been studied by using the Hubbard model [19]. Recently, the density functional theory has been used to determine the electronic and magnetic structure of hydrogen-terminated graphene nano-ribbon edges [20] as well as to study the energy gaps and magnetism in bilayer graphene nano-ribbons in the presence of an external electric field between the layers [21]. Graphenes in the presence of both the 
magnetic and the electric fields have also been studied within a noninteracting tight-binding picture by using the Green function formalism [22]. Few works are also done studying the properties of two and three dimensional graphite lattice structures in the presence of a magnetic field and the on-site Coulomb repulsion [23, 24, 25]. However, a detailed study of the properties of the graphite ribbons with different types of edges, in the presence of both the magnetic field and the Hubbard interaction is yet to be worked out.

In this paper, we investigate how the properties of the graphite ribbons with zigzag and armchair edges vary with the strength of the magnetic field (both parallel and perpendicular to the graphite plane) and the onsite Coulomb repulsion. To understand the role of the special geometry of graphite structures, we also compare our results with similar calculations on the square lattice ribbons. We model the system using the Hubbard Hamiltonian in the presence of a magnetic field, and solve it for the half-filled band within the unrestricted Hartree-Fock Approximation (HFA).

\subsection{The Model}

For convenience, we use a lattice transformation which changes a hexagonal lattice to a brick-type lattice, without changing the lattice topology [4]. Under this transformation the zigzag and the armchair ribbons look like as shown in Fig. 1. We use the following Hamiltonian to describe our system.

$$
H=\sum_{\langle i, j\rangle, \sigma}\left(t_{i, j} c_{i, \sigma}^{\dagger} c_{j, \sigma}+H . c .\right)+U \sum_{i} n_{i, \uparrow} n_{i, \downarrow}-\vec{B} . \sum_{i, \sigma} \vec{\mu}_{\sigma} n_{i, \sigma},
$$

where $c_{i, \sigma}^{\dagger}\left(c_{i, \sigma}\right)$ is the creation(annihilation) operator for an electron with spin $\sigma$ at the $i$-th site. $n_{i, \sigma}=c_{i, \sigma}^{\dagger} c_{i, \sigma}$, and $n_{i}=\sum_{\sigma} n_{i, \sigma}$ is the number operator at the $i$-th site. $t_{i, j}$ is the hopping integral between the $i$-th and the $j$-th sites and $\langle i, j\rangle$ denotes nearest neighbor sites $i$ and $j$. Since we are dealing with an ordered lattice, all $t_{i, j}$ 's are same $(t)$ in the absence of a magnetic field. $U$ is the on-site Coulomb repulsion energy. $\vec{\mu}_{\sigma}$ is the moment of an 
electron with spin $\sigma$ and the magnetic moment is hereafter measured in units of $\mu_{B}$, the Bohr magneton; $\vec{B}$ is the magnetic field. Under the Hartree-Fock Approximation, we decouple the Hubbard term using

$$
U n_{i, \uparrow} n_{i, \downarrow} \rightarrow U\left\langle n_{i, \uparrow}\right\rangle n_{i, \downarrow}+U n_{i, \uparrow}\left\langle n_{i, \downarrow}\right\rangle-U\left\langle n_{i, \uparrow}\right\rangle\left\langle n_{i, \downarrow}\right\rangle,
$$

where the angular brackets denote the expectation values. This leads to two decoupled Hamiltonians for the up and the down spins, which can now be easily diagonalized to obtain the single particle energy levels in a self-consistent manner. This particular approximation has been extensively used in studying short ranged electronic interaction in similar low dimensional systems with reasonable success. Many of the works on graphite systems treated the electronic correlation using the unrestricted HFA calculation which yielded physically reliable phases [3, 17, 18]. Some novel predictions regarding the edge states of the graphenes found in these works are in excellent agreement with the results found by calculations based on the Density Functional Theory (DFT) method [26, 27]. So the unrestricted HFA has already been well tested for the present model at least in the limit $\vec{B}=0$. Moreover, the antiferromagnetic phases driven by the Hubbard correlation in similar low dimensional systems (e.g. one dimensional $\mathrm{C}_{60}$ polymers) has been successfully studied using the same level of approximation [28] to yield a reasonable comparison with experimental findings. Based on the above observations it seems meaningful to explore the ground state properties of our model Hamiltonian (1) using the unrestricted HFA. The stability of the phases obtained in the numerically self-consistent calculation has been checked carefully.

Throughout our study, we consider periodic boundary condition along the length of the ribbon and open boundary condition along its width. One can realize such a boundary condition in practice by wrapping the ribbon in the form of a cylinder whose axis is parallel to its width. However, we would like to stress that in our study we have considered the flat ribbon under periodic boundary condition along its length. As we shall show later on, the results become insensitive to the boundary condition for system length considered in our work. We have studied the ribbons of different lengths $(50-150)$ and 
widths $(3-8)$. For the sake of comparison we have presented the results for the systems with the same length (150); the widths are varied as per requirement. We consider two separate cases, $(i)$ magnetic field parallel to the ribbon plane, and $(i i)$ magnetic field perpendicular to the ribbon plane. When the magnetic field $\vec{B}$ is parallel to the ribbon plane, all nearest neighbor hopping terms along the length of the ribbon will be modified by the same Peierl's phase $\phi$, such that

$$
\begin{aligned}
& t_{i, j} \rightarrow t e^{2 \pi i \phi / \phi_{0}}, \\
& \phi=\frac{1}{N} \int \vec{B} \cdot \overrightarrow{d S},
\end{aligned}
$$

where the surface integral is carried over the cross section of the cylinder. $N$ is the length of the ribbon in units of lattice constant. In the second case, when the magnetic field $\vec{B}$ is perpendicular to the ribbon plane, we choose the Landau gauge $\vec{A}=(0, B x, 0)$, where we have assumed the $Y$ axis to be along the translationally invariant direction and the $X$ axis perpendicular to the $Y$ axis in the ribbon plane. Now the nearest neighbor hopping integrals along the length of the ribbon will depend on the $X$ coordinate or the layer number in the following way,

$$
t_{i, j} \rightarrow t e^{2 \pi i \phi_{i, j} / \phi_{0}} \quad, \quad \phi_{i, j}=\int_{i}^{j} \vec{A} \cdot \overrightarrow{d l}
$$

which lead to

$$
\phi_{i, j}=\frac{\phi}{2} m \quad \text { for graphite ribbon, }
$$

and

$$
\phi_{i, j}=\phi m \quad \text { for square lattice ribbon, }
$$

where $\phi$ is the flux through a plaquette and $\phi_{0}$ is the flux quantum; $m$ denotes the layer number of the ribbon containing the $i$-th and the $j$-th sites. While performing the transformation of the lattice one ensures that the flux through one basic hexagonal plaquette in the graphene equals the flux through one basic rectangular plaquette in the brick-type lattice. This restriction determines the nearest neighbor distance $a$ in the brick-type lattice in terms of the nearest neighbor distance $b$ in the graphene ribbon. We use a typical value 
for the latter: $b=.25 \mathrm{~nm}$ [29]. In all of our calculation we set the scale of energy by setting the nearest neighbor hopping integral $t=1.0$.

For the purpose of illustration we consider the case of $\mu_{B} B / t=1$. For the graphite ribbons $t$ is $2.7 \mathrm{ev}$ [30]. Value of the magnetic field $B$ in this situation is $4.6 \times 10^{4}$ Tesla.

We calculate the density of states $\rho(E)=\frac{1}{N} \sum_{i} \delta\left(E-E_{i}\right)$, where $E_{i}$ 's are the energy eigenvalues. To study the conductivity of the system, we calculate the Drude weight $(D)$ and the charge gap $(\Delta)$. The charge gap $(\Delta)$ at the Fermi level of a system containing $n$ electrons is given by

$$
\Delta=E_{n+1}+E_{n-1}-2 E_{n}
$$

where $E_{n}$ is the ground state energy for a system of $n$ electrons. To calculate the Drude weight $(D)$, a vanishingly small magnetic flux $\phi^{\prime}$ is introduced along the axis of the cylinder-shaped ribbon. The flux $\phi^{\prime}$ does not penetrate the ribbon, so it does not alter the Zeeman interaction term and only modifies the hopping integrals parallel to the length of the ribbon according to Eq. 3. The Drude weight is calculated from the formula [31, 32]

$$
D=\frac{N}{4 \pi^{2}}\left(\frac{\partial^{2} E\left(\phi^{\prime}\right)}{\partial \phi^{\prime 2}}\right)_{\phi^{\prime}=0}
$$

where $E\left(\phi^{\prime}\right)$ is the ground state energy of the system in the presence of the flux $\phi^{\prime}$.

\subsection{Magnetic field parallel to the graphite plane}

It can be shown analytically that in the absence of the Hubbard interaction and the magnetic field, the armchair ribbons with widths $3 M$ and $3 M+1$ are insulating and ribbons with width $3 M-1$ are conducting ( $M$ is any integer) at half-filling [4, 33], we shall present here one case of width $3 M-1$ (width 5: $M=2$ ) and one case of width $3 M$ (width $3: M=1$ ). Cases with large 
values of $M$ have been investigated and are found to yield results which are similar to the ones that have been presented here. In Fig. 2, we show the density of states for the armchair ribbons with widths 3 and 5 for $U=0$ and $U>0$ in the absence of the magnetic field. Since at $B=0$, the up and the down spin bands are degenerate, these diagrams represent the density of states for both the bands. It is clear from the diagrams that at $U=0$, there is a gap at the Fermi level for the armchair ribbon with width 3 at half-filling; this indicates an insulating behavior, while there is no such gap for width 5 indicating a metallic or conducting behavior. These show that our numerical results are in agreement with the analytic results for the armchair ribbons in the absence of the magnetic field and the Hubbard correlation.

For $U>0$, we observe a few gaps in the energy spectrum other than the Hubbard gap. These gaps are seen for small ranges of values of $U$, listed below :

$$
\begin{array}{ll}
1.8 \leq U \leq 3.6 & \text { for width } 3 \\
3.3 \leq U \leq 4.1 & \text { for width } 5
\end{array}
$$

We have presented the cases with $U=3$ and $U=3.6$ for armchair ribbons with widths 3 and 5 respectively. These gaps arise due to the special geometry of the graphite ribbons. It was observed that the position and the size of these gaps depend on the width of the systems and the magnitude of the Hubbard interaction.

Now as we turn on the magnetic field parallel to the graphite plane, spin reversal symmetry breaks down. The up and the down spin bands are now non-degenerate. The number of up spins increases in the system at the cost of the down spins. In the diagram of the density of states, the only change is the shift of the whole spectrum in the energy scale; it moves towards left for the up spin bands and towards right for the down spin bands. The amount of shift depends only on the magnitude of the applied magnetic field. So it is clear from the diagrams that for certain values of the magnetic field, the Fermi level will lie within one of the gaps resulting in insulating phases and 
for other values of it the system will be conducting. For high enough values of the magnetic field the system consists of up spins only and it becomes spin polarized.

In the absence of the magnetic field and the Hubbard correlation, analytic calculations show that the zigzag ribbons are metallic at half-filling [4, 33]. In Fig. 3, we present the density of states for zigzag ribbon with width 3 both in the absence and in the presence of the Hubbard interaction. It shows that the energy spectrum is gapless for $U=0$ (Fig. 3(a)) and for $U>0$ there is only one gap at the Fermi level (Fig. 3(b)) which is nothing but the Hubbard gap.

The Drude weight $(D)$ and the charge gap $(\Delta)$ give a clear idea of the conductivity of the system. Drude weight becomes high in the conducting region and zero in the insulating region, while the charge gap is nonzero in the insulating region and becomes zero or of the order of the inverse of the system size in the conducting region. Variations of the Drude weight and the charge gap with the applied magnetic field for a few fixed values of the Hubbard interaction parameter are shown in Fig. 4. First two diagrams represent the cases for the armchair ribbons with widths 3 and 5 respectively and the third one shows the case for the zigzag ribbon with width 3 . Though we have presented the variations for a few values of the Hubbard interaction parameter, similar kind of behavior is observed also for other values of it.

Using the data for the density of states, the Drude weight and the charge gap, we have constructed the phase diagrams for these systems in the $U-B$ plane, which give a detailed idea of the conduction property of the systems. In Fig. $5(a)$ and $5(b)$, we have drawn the $U-B$ phase diagrams of the armchair ribbons with widths 3 and 5 respectively. It is clear from these phase diagrams that for $U=0$, the system is insulating for width 3 and conducting for width 5 in absence of magnetic field. As the magnetic field is turned on and increased, after a critical value of $B$, the armchair ribbon with width 3 becomes conducting. On the other hand the ribbon with width 5 continues 
to be conducting in the presence of $B$. In absence of the magnetic field the system is insulating due to the Hubbard gap in the spectrum for $U>0$. A finite value of the magnetic field is required to turn the system conducting. At moderate values of $U$ (the range specified in Eq. 9), with the increase of $B$, two additional phase transitions are observed for both the cases, one from a metallic to an insulating phase and another from an insulating to a metallic phase. As a result of this, small insulating portions are observed in the middle of the conducting regions of the phase diagrams. These insulating islands arise due to the gaps other than the Hubbard gaps shown in the plot of the density of states (Fig. 2). These insulating lobes decrease in size with the increasing width. It is to be noted here that an increase in the system length (even by an order of magnitude) does not change the positions and the sizes of the lobes. In fact the results that we have presented here for system length $N=150$ have already become independent of the length of the system, and thus practically independent of the boundary condition imposed on the system. At a large value of $B$, the up spin band of the system becomes completely filled and the down spin band completely empty - resulting in an insulating situation for both types of armchair ribbons. The critical value of $B$ for which the system assumes the spin polarized phase becomes smaller and smaller with the increase of $U$, because an enhancement of Hubbard correlation increases the Hubbard gap enormously and greatly reduces the band width. Though we have not shown the diagrams for armchair ribbons with width 4 (or $3 M+1)$, we have studied it and observed that its behavior is qualitatively similar to that of width 3 .

Figure 5(c) represents the $U-B$ phase diagram for a zigzag ribbon with width 3 . For $U=0$ the zigzag ribbon is conducting as there appears no gap at the Fermi level. Nonzero $U$ opens a gap at the Fermi level making the system insulating. For a small value of $U$, only a small value of $B$ is sufficient to drive the system conducting, while a higher value of $U$ requires a higher value of $B$ for the transition. As we have seen in the case of the armchair ribbons, in this case also a high enough value of $B$ makes the system spin polarized. So for the zigzag ribbons, application of magnetic field parallel 
to the graphite plane causes one phase transition from an insulating to a conducting one and another from a conducting to an insulating spin polarized one for nonzero values of $U$. In this case, no other insulating lobe at the middle of the conducting region is obtained, since there is no gap in the energy spectrum other than the Hubbard gap.

This is interesting to note that only in the case of the armchair ribbon there arise additional gaps apart from the usual Hubbard gap (see, for example, the gap around $E=3.5$ in Fig. 2(b)). In the presence of the Hubbard correlation (i.e. $U \neq 0$ ) the system is driven to a spin density wave (SDW) state. If one moves along the edge of the ribbon one should encounter a distribution of moments corresponding to a dominant SDW with wave vector $q=\pi$. This leads to the formation of up and down spin moments at the neighboring sites which look like a one dimensional antiferromagnetic modulation. It is well known that such an SDW modulation is responsible for opening up Mott-Hubbard gap at the Fermi level of a half-filled electronic band in a bipartite lattice. However, in the case of an armchair ribbon we find an additional underlying modulation of moments corresponding to an SDW with $q \neq \pi$. It can be traced by observing the repetition of the peaks of $S_{i}$ of same height in Fig. 6(a). This type of modulation is not observable in zigzag ribbons (Fig. 6(b)). The additional SDW modulation is responsible for opening up additional gaps in the spectrum of the armchair ribbons in case of $U \neq 0$. Certainly these gaps will decrease with increasing ribbon width but will remain sensibly unchanged with increasing length. As we have noted in Eq. 9 these gaps are observable for a narrow range of value of $U$ that depends on the width of the system. Larger $U$ values wipe out the effect of the additional SDW modulation and consequently the usual Mott-Hubbard gap alone survives.

We check the sensitivity of our results to the system length by calculating a quantity $\Sigma$ defined by $\Sigma=\left[\sum_{i=1}^{N} \frac{\left(\sigma_{i}^{\mathrm{pbc}}-\sigma_{i}^{\mathrm{obc}}\right)^{2}}{N}\right]^{\frac{1}{2}}$, where $\sigma_{i}=n_{i, \uparrow}-n_{i, \downarrow}$, the moment at site $i$ along the symmetry axis of the ribbon. The variation of 
$\Sigma$ with the system length $N$ is plotted in Fig. $6(c)$ for a typical value of $U$ $(=2.4)$ and $B(=1.0)$. It turns out that the results become insensitive to the boundary condition as $\Sigma$ goes to zero for $N \sim 100-150$.

\subsection{Magnetic field perpendicular to the graphite plane}

In this section similar studies are done but the direction of the magnetic field is taken perpendicular to the ribbon plane. The effect of the magnetic field $B$ is quite complicated in this case. As we have discussed earlier (Eqs. 4 and 5 ), for this type of magnetic field the hopping integrals in different layers are modified differently. It causes the formation of multiple bands (depending on the magnitude of the applied magnetic field) in the energy spectrum. On the other hand, the Zeeman term present in the Hamiltonian shifts the energy spectrum for the up and down spin bands in opposite directions. These two processes together contribute to the properties of the system. In Fig. 7, we show the density of states of the armchair ribbons with widths 3 and 5 for $U=0$ in the presence of the magnetic field $B$. For both $U=0$ and $B=0$, we have seen the nature of the density of states earlier in Fig. 2. Here we see that for nonzero $B$, gaps open at different parts of the energy spectrum. We have shown the case with $B=1.65$. Even for $U>0$ this type of gaps are observed. In Fig. 8, the density of states for finite $U$ is shown for the same value of the magnetic field.

Next we present the cases of the zigzag ribbons with width 3 . Figures $9(a)$ and $9(b)$ show the nature of the density of states for $U=0$ and $B=1.65$. In this case also multiple gaps appear in the energy spectrum. Figures $9(c)$ and $9(d)$ show the same for $U=2$.

With the magnetic field perpendicular to the ribbon plane, the positions of the gaps in the energy spectrum depend on the magnitude of the magnetic field, unlike the case with the magnetic field parallel to the ribbon plane. Studying the variations of the Drude weight and the charge gap with $B$ for 
fixed $U$ values, we see that a few transitions occur between the insulating and the conducting phases with the increase of $B$ from the zero value. Figure 10 shows one representative case (for armchair ribbon of width 3 at $U=1$ ) of such variations.

To get a clear view, we have plotted the $U-B$ phase diagrams of the systems in Fig. 11. Here the first two diagrams are for the armchair ribbons with widths 3 and 5 respectively and the third one is the same for the zigzag ribbon of width 3 . For the purpose of comparison we have also presented the phase diagram for the square lattice ribbon in the same panel. It shows that for a fixed value of $U$ (weak to intermediate coupling) there appear small domains along the vertical direction (width depending on the value of $U$ ) where the system becomes metallic. Thus we find some conducting lobes immersed in the "sea" of a large insulating region. These metallic lobes are marked with even Roman numerals (e.g. II, IV and VI) in Fig. 11. Apart from these there also appear tiny metallic lobes in the case of the armchair ribbon of width 5 (within the insulating region $\mathrm{V}$ in Fig. 11(b)). In the case of the zigzag ribbon, on the contrary, we find a very small insulating lobe inside a conducting one (i.e. lobe number IV in Fig. 11(c)). Such tiny lobes are observed to be finite size effects and generally disappear with increasing length/ width of the system. It is to be noted here that within these metallic regions there appears a partial spin polarization that increases with the increase in the magnetic field strength. Due to the existence of multiple metallic lobes one can observe, in principle, several Metal-Insulator (MI) transitions driven by the external magnetic field. Observing first one or two of such transitions may be experimentally feasible in the case of the armchair configuration, although, in general, the value of $B$ required to see all these transitions will be too high compared to the present experimental facility. It is seen from Figs. 11(a) and 11(b) that in the case of armchair ribbons, for large enough $U$, the width of the conducting lobe (region II) is so small that in this region two consecutive MI transitions may take place with a small increase in $B$, a feature that may be interesting in view of some field-driven switching mechanisms. 
On the other hand the insulating region shows a rich variety of phase structure controlled by the values of the parameters as well as crucially controlled by the boundary effects and system widths. Generally, after a large enough value of $B$ the system becomes spin polarized (e.g. region $\mathrm{V}$ in Figs. 11(a)-(c)) beyond which no further phase transitions occur with increasing $B$. The first insulating region (region I in Figs. 11(a)-(c)) obtained for small values of $B$ shows some magnetic structure for $U \neq 0$. For the armchair ribbons this region shows an antiferromagnetic modulation of electron spins in a given layer. However, this antiferromagnetic phase is not homogeneous as usually observed due to a Hubbard interaction on an infinite bipartite lattice. Here, by moving from one layer to an adjacent layer, one finds that the sublattice magnetization per layer strongly depends on the layer index (controlled essentially by the proximity of the boundary). For a ribbon with odd number of layers $(3,5$ or 7$)$ there always appears an "inversion" symmetry of the patterns about the central layer. For ribbons of even number of layers too such a symmetry exists about the central horizontal axis (passing through midway between two middle layers of atoms). In case of a zigzag ribbon, however, this insulating region shows an antiferrimagnetic alignment along the layers at or near the boundary, and a perfect antiferromagnetic alignment only at the central layer (for odd width). The reason behind this discrepancy between the zigzag and the armchair configuration is much similar to that explained in the context of Fig. 6. The other insulating patch that is interspersed between the conducting lobes (e.g. region III) have partial spin polarization forming a net magnetization in the system (due to an appreciably large value of $B$ ). In the case of armchair ribbons one finds a spin distribution much like a ferro-arrangement, magnetization in a layer decreasing from the boundary to the central layer (obeying the previously mentioned inversion symmetry). In the case of the zigzag ribbon the moment distribution pattern is "ferrimagnetic" in a layer. Again the variation of the moment distribution from the boundary layer to the central one shows the aforesaid "inversion" symmetry. This shows that in the presence of a magnetic field the same hexagonal network manifests the effect of Coulomb 
correlation in different ways subject to the presence of boundaries of different geometries at finite distances.

In the special case of an armchair ribbon of width 3 the insulating region I persists even upto $U=0$ (known to be an exact result at least for $B=0$ [4, 33]) where the system behaves like a paramagnetic insulator. Antiferromagnetism is switched on as soon as $U$ becomes non-zero. For the armchair ribbon of width 5 , however, the system is conducting at $U=0, B=0$. Antiferromagnetism sets in for $U>0$ in the absence of the magnetic field. In this later case the antiferromagnetic order parameter shows a sudden increase in its value around $U / t \sim 2.2$ for $B=0$. However, within the present mean field calculations one cannot predict a critical value of $U / t$, even if it is there, because the charge gap and the antiferromagnetic order parameter truly vanish only at $U=0$.

\subsection{Comparison between the graphite and the square lattice ribbons}

To understand the effect of the special type of structures of graphite lattices, we have studied for comparison the square lattice ribbon structures of width 3 at half-filling. These systems are metallic in the absence of the Hubbard interaction and the magnetic field. Non-zero Hubbard interaction makes the system insulating. It is clear from the plot of the density of states with $B=0$ (Fig. 12) that the $U-B$ phase diagram (when $B$ is parallel to the ribbon plane) will be qualitatively similar to that of the zigzag graphene ribbons. In Fig. 13, the density of states of the square lattice ribbons of width 3 in the presence of the magnetic field perpendicular to the ribbon plane is presented. Here also multiple gaps open up in the energy spectrum. Fig. 11(d) shows the $U-B$ phase diagram for this case. From Fig. 11 it is revealed that the phase diagram of the square ribbon resembles closely that of the armchair graphene rather than the that of the zigzag ribbon. Also the nature of spin polarization in the insulating regions for the square lattice is found to be much similar to the case of the armchair ribbons. In view of this above mentioned similarity between the armchair and square lattice ribbons we further 
note that the sites on the boundary layers of each of these ribbons belong to the similar status with respect to the coordination numbers. On the other hand the sites of the boundary layer of a zigzag ribbon are not at the same status (see Fig.1).

\subsection{Conclusion}

We have studied the simultaneous effects of the magnetic field and the on-site Coulomb interaction on the nanographite ribbon structures. Single orbital nearest neighbor tight-binding model has been used to study the systems. Electronic correlation is included in the Hamiltonian using the Hubbard interaction. Reasonably large systems are studied in this work. We have used the unrestricted Hartree-Fock Approximation method, which was found to be reliable in these type of systems [3, 17, 18]. Since a magnetic field in any direction can be resolved into two components, one parallel and another perpendicular to the graphite plane, we have studied the effects of the magnetic fields in the two above mentioned directions independently. In nanographite structures, shapes of the boundary play an important role in determining the behavior of the systems. Here we have considered two important boundary geometries - the zigzag and the armchair, and studied the effect of the magnetic field on the ribbon structures under periodic boundary condition along their lengths. We have calculated the density of states for different $U$ and $B$ values. Studying these density of states, the charge gap and the Drude weight we obtained the $U-B$ phase diagrams for different types of graphite ribbons. To get a clear understanding of the geometry effect, we have compared the results of the graphite ribbons with that of the square lattice ribbons. With the magnetic field parallel to the ribbon plane these phase diagrams show that the behavior of the armchair ribbons is markedly different from those of the zigzag and the square lattice ribbons. For armchair ribbons small insulating islands at the middle of the conducting regions of the phase diagrams are obtained which are absent in other cases. The reason behind this difference in behavior of armchair and zigzag ribbons is also qualitatively ex- 
plained. A close comparison between the case of armchair and zigzag ribbons in the presence of a magnetic field reveals that the same hexagonal graphene network manifests the effect of the Hubbard interaction in different ways controlled by the boundary topology. When the magnetic field is perpendicular to the ribbon plane, the phase diagram contains several conducting lobes submerged in an insulating sea. Therefore, such systems would show multiple metal-insulator transitions tuned by the magnetic field although much higher values of the field would be required to see these transitions than are commonly realizable in the laboratories at present. In view of such multiple metal-insulator transitions the graphite ribbons may find their practical application in designing field-driven switching devices. Further study of the graphite ribbons with other boundary geometries may yield some interesting results. Also the effect of the finite temperature on the graphite ribbons for the competing regime of the correlation parameter and the magnetic field may be interesting.

\section{acknowledgments:}

One of the authors (BB) was supported by the Jadavpur University Research Grant from the Jadavpur University, Kolkata. 


\section{Bibliography}

[1] S. Ijima, Nature (London) 354, 56 (1991)

[2] K. Nakada, M. Fujita, G Dresselhaus, and M. S. Dresselhaus, Phys. Rev. B 54, 17954 (1996)

[3] M. Fujita, K.Wakabayashi, K. Nakada, and K. Kusakabe, J. Phys. Soc. Jpn. 65, 1920 (1996)

[4] K. Wakabayashi, M. Fujita, H. Ajiki, and M. Sigrist, Phys. Rev. B 59, 8271 (1999)

[5] N. Hamada, S. Sawada, and A. Oshiyama, Phys. Rev. Lett. 68, 1579 (1992)

[6] C. T. White, D. H. Robertson, and J. W. Mintmire, Phys. Rev. B 47, 5485 (1993)

[7] J. W. Mintmire, B. I. Dunlap, and C. T. White, Phys. Rev. Lett. 68, $631(1992)$

[8] J. W. G. Wildöer, L. C. Venema, A. G. Rinzler, R. E. Smalley, and C. Dekker, Nature(London) 391, 59 (1998)

[9] T. W. Odom, J. L. Huang, P. Kim, and C. M. Lieber, Nature(London) 391, 62 (1998)

[10] A. H. C. Neto, F. Guinea, N. M. R. Peres, K. S. Novoselov, and A. K. Geim, Rev. Mod. Phys. 81, 109 (2009) 
[11] K. S. Novoselov, A. K. Geim, S. V. Morozov, D. Jiang, Y. Zhang, S. V. Dubonos, I. V. Grigorieva, and A. A. Firsov, Science 306, 666 (2004); K. S. Novoselov, A. K. Geim, S. V. Morozov, D. Jiang, M. I. Katsnelson, I. V. Grigorieva, S. V. Dubonos, and A. A. Firsov, Nature 438, 197 (2005)

[12] Y. Zhang, Y. Tan, H. L. Stormer, and P. Kim, Nature(London) 438, $201(2005)$

[13] J. P. Lu, Phys. Rev. Lett. 74, 1123 (1995)

[14] H. Ajiki, and T. Ando, J. Phys. Soc. Jpn. 62, 1255 (1993)

[15] M. F. Lin, and K. W. K. Shung, Phys. Rev. B 51, 7592 (1995)

[16] A. Bachtold, C. Strunk, J. P. Salvetat, J. M. Bonard, L. Forro, T. Nussbaumer, and C. Schönenberger, Nature(London) 397, 673 (1999)

[17] A. Yamashiro, Y. Shimoi, K. Harigaya, and K. Wakabayashi, Phys. Rev. B 68, 193410 (2003)

[18] A. Yamashiro, Y. Shimoi, K. Harigaya, and K. Wakabayashi, Physica E 22, $688(2004)$

[19] J. J. Palacios, J. Fernandez-Rossier, and L. Brey, Phys. Rev. B 77, 195428 (2008)

[20] T. Wassmann, A. P. Seitsonen, A. Marco Saitta, M. Lazzeri, and F. Mauri, Phys. Rev. Lett. 101, 096402 (2008)

[21] B. Sahu, H. Min, A. H. MacDonald, and S. K. Banerjee, Phys. Rev. B 78, $045404(2008)$

[22] C. Ritter, S. S. Makler, and A. Latge, Phys. Rev. B 77, 195443 (2008)

[23] I. F. Herbut, Phys. Rev. Lett. 97, 146401 (2006)

[24] I. F. Herbut, Phys. Rev. B, 76, 085432 (2007) 
[25] N. M. R. Peres, M. A. N. Araujo, and D. Bozi, Phys. Rev. B 70, 195122 (2004)

[26] S. Okada, and A. Oshiyama, Phys. Rev. Lett 87, 146803 (2001)

[27] H. Lee, Y. Son, N. Park, S. Han, and J. Yu, Phys. Rev. B 72, 174431 (2005)

[28] K. Harigaya, Phys. Rev. B 53, R4197 (1996)

[29] Aketagawa M., Ikeda Y., Tanyarat N. and Ishige M., Meas. Sci. Technol 18, $503(2007)$

[30] Yazyev O. V., Phys. Rev. Lett 101, 037203 (2008)

[31] W. Kohn, Phys. Rev. 133, A171 (1964)

[32] R. M. Fye, M. J. Martins, D. J. Scalapino, J. Wagner, and W. Hanke Phys. Rev. B44, 6909 (1991)

[33] S. Nakamura, K. Wakabayashi, A. Yamashiro, and K. Harigaya, Physica E 22, 684 (2004) 


\section{Figure captions}

Fig 1: The transformation from hexagonal to brick-type lattice, without changing the lattice topology.

Fig 2: Plot of the density of states (D.O.S) of the armchair ribbons of length 150 in absence of magnetic field. The widths (w) of the ribbons and the magnitude of the Hubbard interaction $(U)$ are specified on the diagrams.

Fig 3: Plot of the density of states of the zigzag ribbons of length 150 in absence of magnetic field. The widths (w) of the ribbons and the magnitude of the Hubbard interaction $(U)$ are specified on the diagrams.

Fig 4: Plot of the Drude weight $(D)$ and the charge gap $(\Delta)$ as functions of magnetic field (parallel to ribbon plane) for the armchair and the zigzag graphite ribbons (length 150) at half-filling; dotted line is for the Drude weight and solid line is for the charge gap. The type and the widths (w) of the ribbons and also the magnitude of the Hubbard interaction $(U)$ are specified on the diagrams.

Fig 5: $U-B$ phase diagrams of the armchair and the zigzag ribbons of length 150 when the magnetic field is parallel to the graphite plane at half-filling. The widths (w) and the type of the ribbons are mentioned on the diagrams. The "ins" in the second diagram means insulating region.

Fig 6: Plot of $S_{i}=n_{i \uparrow}-n_{i \downarrow}$, the moment at site $i$ along the upper edge, as a function of the site index $i$ for graphite ribbons with (a) armchair boundary and (b) zigzag boundary at $U=3$. and $B=0$.. For simplicity we consider ribbons with width 3 and length 20. Features are similar for larger systems. Results given in (b) closely resemble those found using DFT calculations [26, 27]. In $(c)$ we have plotted $\Sigma$ as a function of system length $N$. This graph checks the insensitivity of our result to the boundary condition for our system length 150 . 
Fig 7: Plot of the density of states of the armchair ribbons of length 150 in presence of magnetic field $\left(\mu_{B} B / t=1.65\right)$ perpendicular to graphite plane when $U / t=0$. Widths ( $\mathrm{w}$ ) of the ribbons are specified on the diagrams.

Fig 8: Plot of the density of states of the armchair ribbons of length 150 in presence of magnetic field $\left(\mu_{B} B / t=1.65\right)$ perpendicular to graphite plane and Hubbard interaction $(U / t=2)$. Widths $(\mathrm{w})$ of the ribbons are specified on the diagrams.

Fig 9: Plot of the density of states of the zigzag ribbons of length 150 and width 3 in presence of magnetic field $\left(\mu_{B} B / t=1.65\right)$ perpendicular to the graphite plane. On-site Coulomb correlation parameter values are mentioned on the diagrams.

Fig 10: Plot of the Drude weight $(D)$ and the charge gap $(\Delta)$ as functions of magnetic field (perpendicular to ribbon plane) for the armchair graphite ribbon of length 150 and width 3 at half-filling; dashed line is for the Drude weight and solid line is for the charge gap. The magnitude of the Hubbard interaction $(U)$ is 1 .

Fig 11: $U-B$ phase diagrams of armchair ((a) and (b) for widths 3 and 5 respectively), zigzag ((c) for width 3$)$ and square lattice ribbons ((d) for width 3) of length 150 when the magnetic field is perpendicular to the ribbon plane, at half-filling.

Fig12: Plot of the density of states of the square lattice ribbons of width 3 and length 150 in absence of magnetic field. On-site Coulomb correlation parameter values are mentioned on the diagrams.

Fig 13: Plot of the density of states of the square lattice ribbons of length 150 and width 3 in presence of magnetic field $(B=1.65)$ perpendicular to ribbon plane. On-site Coulomb correlation parameter values are mentioned on the diagrams. 


\section{Figures}
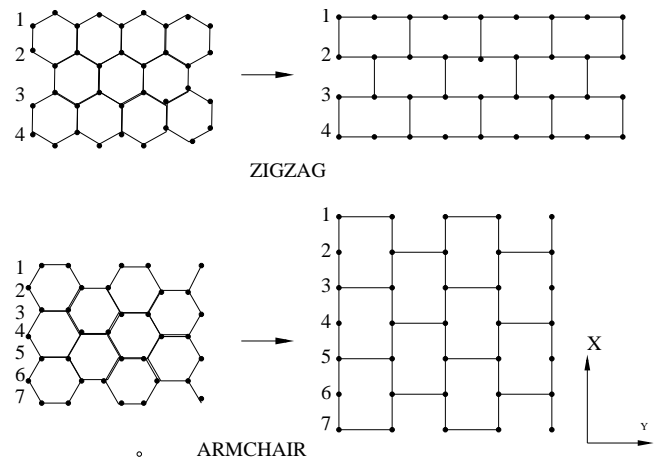

Figure 1: 

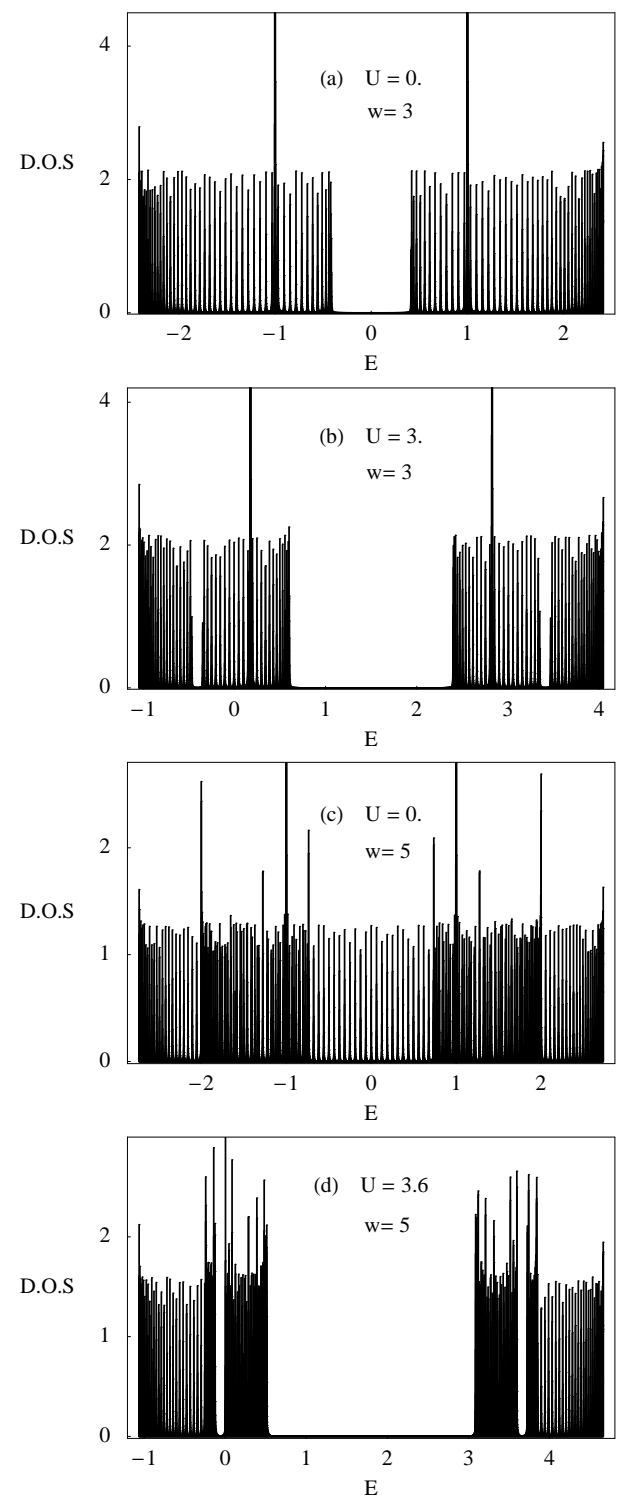

Figure 2: 

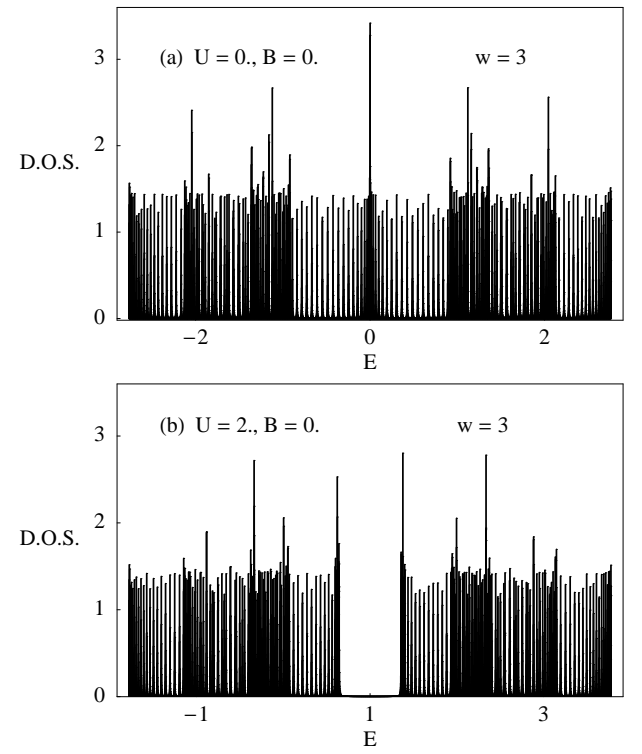

Figure 3: 

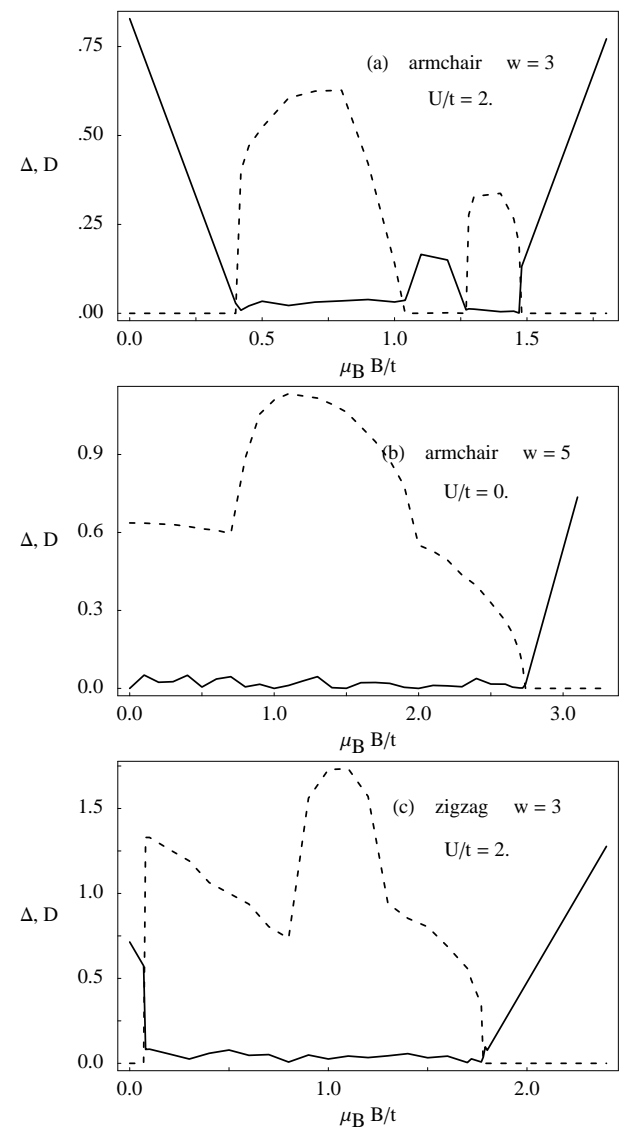

Figure 4: 

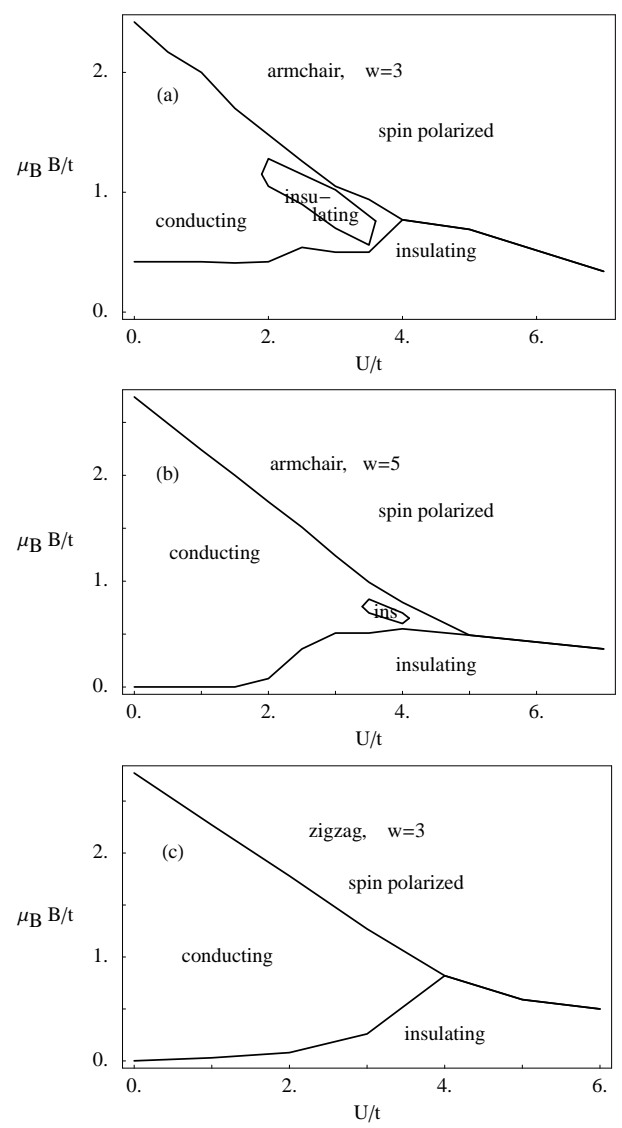

Figure 5: 

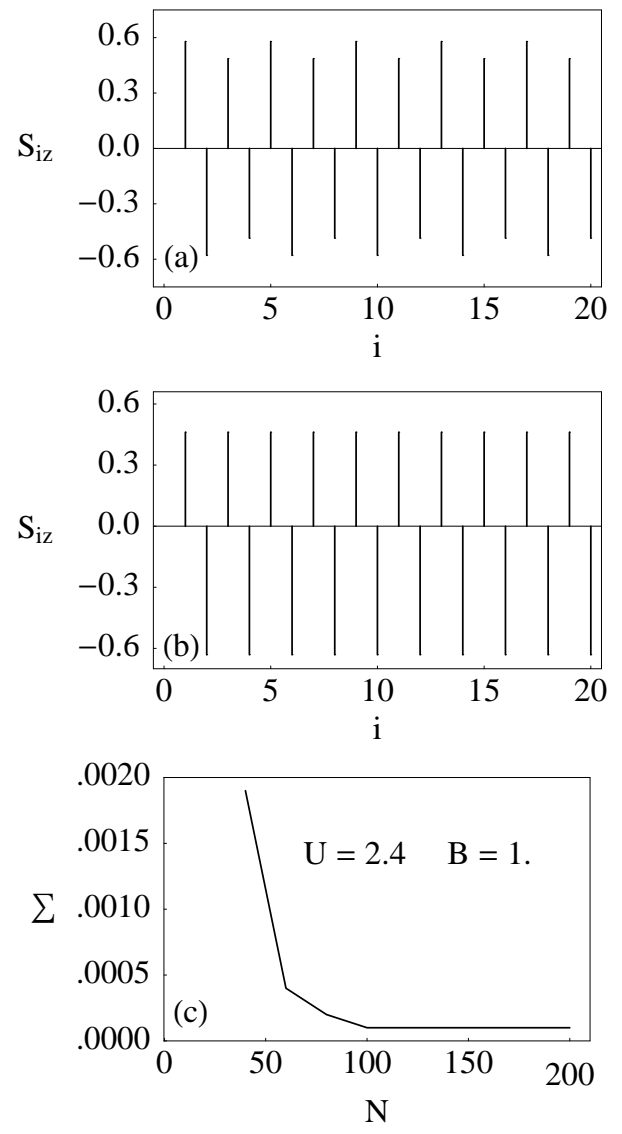

Figure 6:

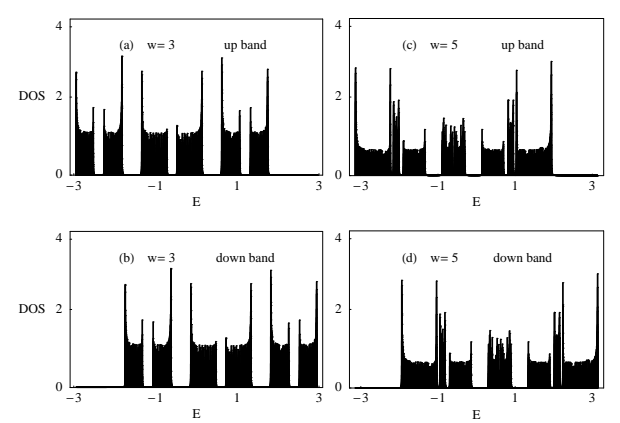

Figure 7: 

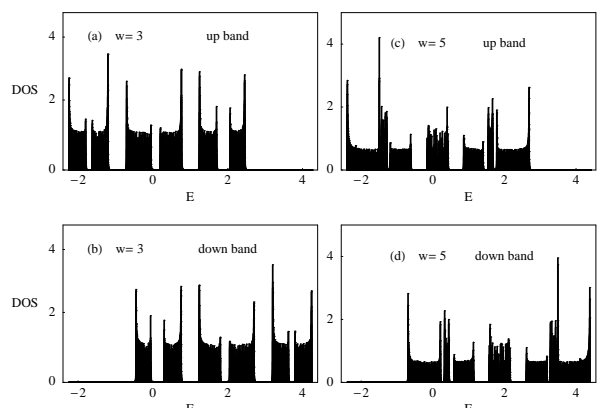

Figure 8:

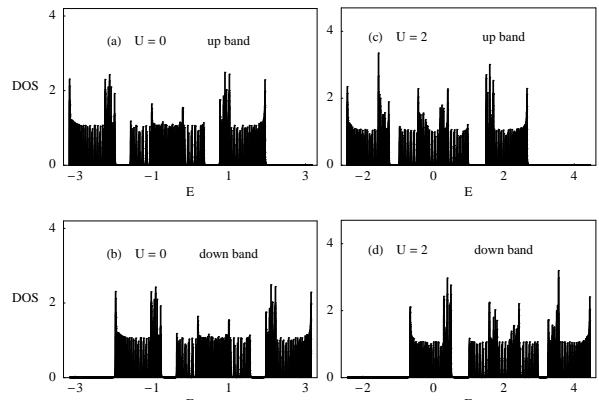

Figure 9:

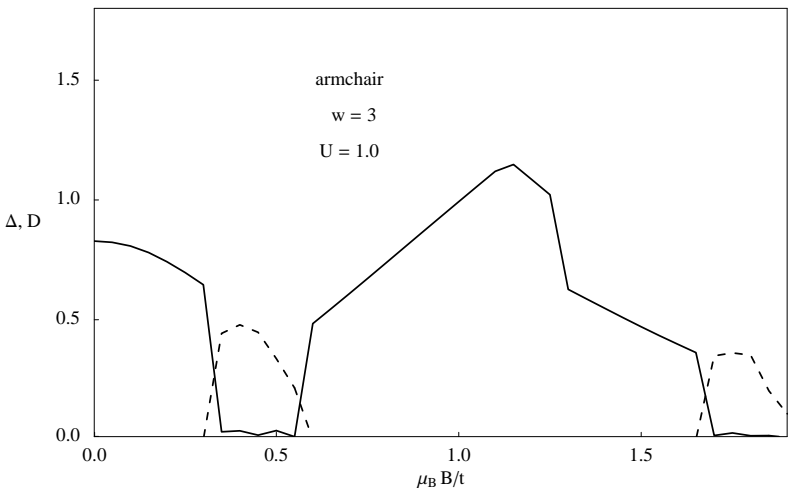

Figure 10: 

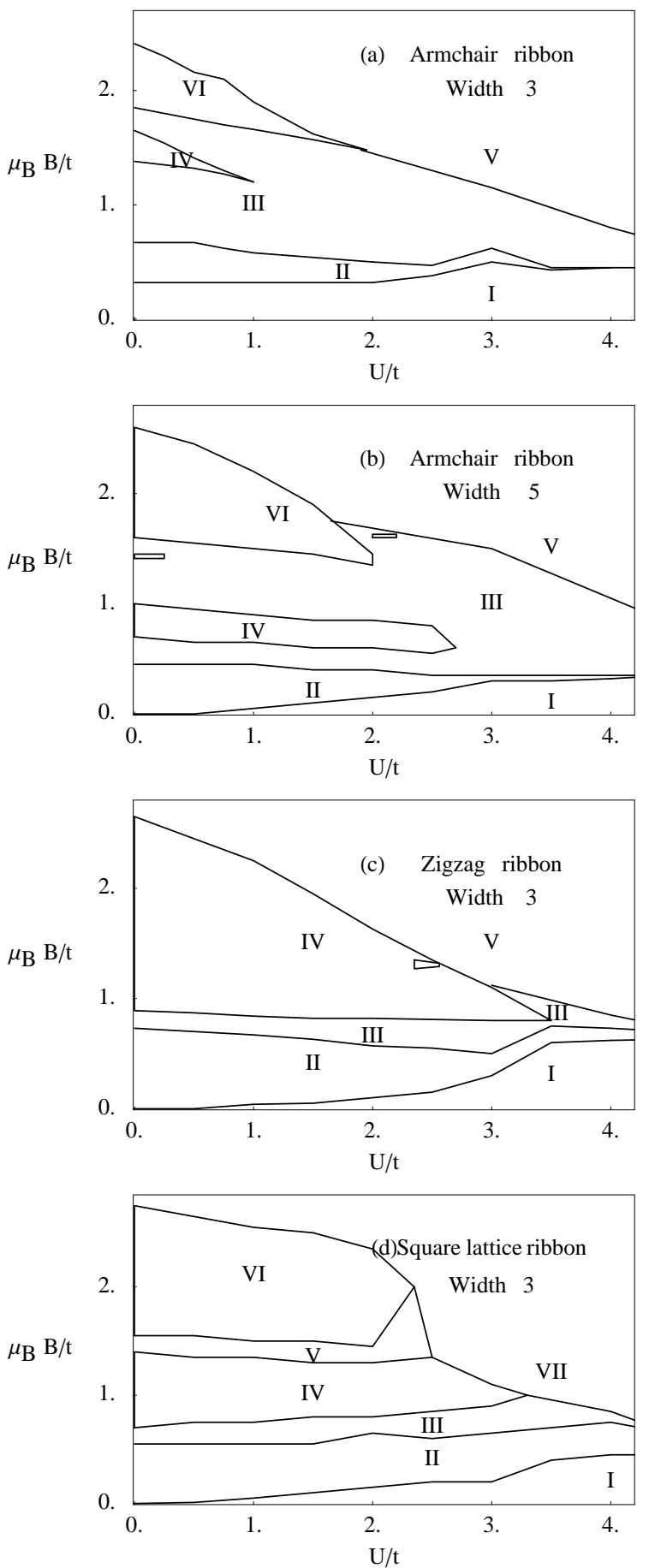

Figure 11: 

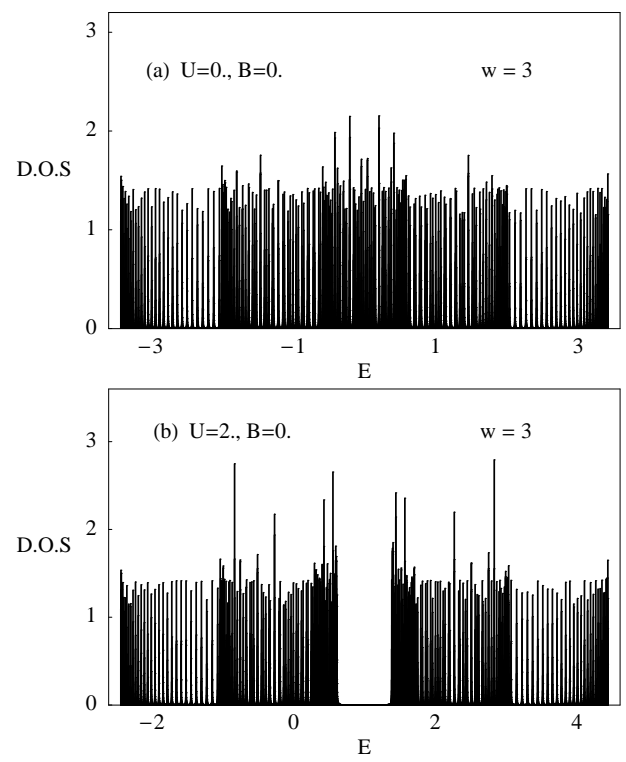

Figure 12:
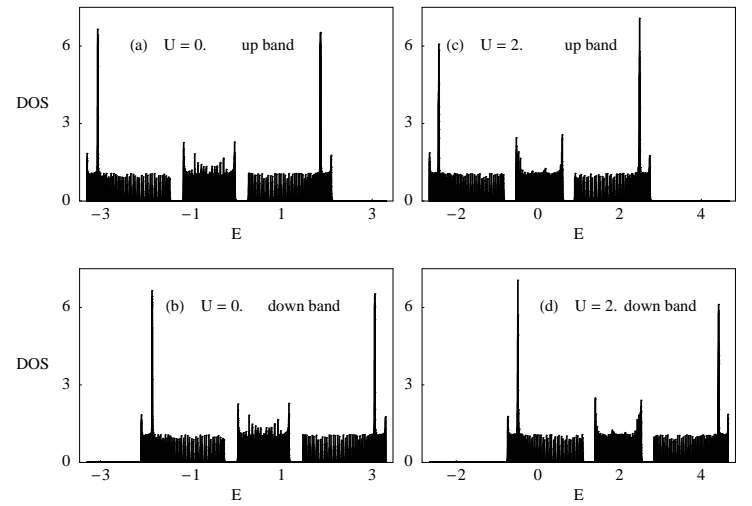

Figure 13: 